\title{
Role of Transient Receptor Potential Vanilloid 4 in Neutrophil Activation and Acute Lung Injury
}

Jun Yin ${ }^{1,2 *}$, Laura Michalick ${ }^{3 *}$, Christine Tang ${ }^{2,4 *}$, Arata Tabuchi ${ }^{2}$, Neil Goldenberg ${ }^{2}$, Qinghong Dan ${ }^{2}$, Khader Awwad $^{5}$, Liming Wang ${ }^{1,2}$, Lasti Erfinanda ${ }^{3}$, Geraldine Nouailles ${ }^{6}$, Martin Witzenrath ${ }^{6}$, Alexis Vogelzang ${ }^{7}$, Lu Lv ${ }^{1}$, Warren L. Lee ${ }^{2}$, Haibo Zhang ${ }^{2}$, Ori Rotstein ${ }^{2,8}$, Andras Kapus ${ }^{2,8}$, Katalin Szaszi ${ }^{2,8}$, Ingrid Fleming ${ }^{5}$, Wolfgang B. Liedtke ${ }^{9}$, Hermann Kuppe ${ }^{10}$, and Wolfgang M. Kuebler ${ }^{2,3,8,10}$

${ }^{1}$ Department of Cardiothoracic Surgery, Affiliated People's Hospital of Jiangsu University, Zhenjiang, China; ${ }^{2}$ Keenan Research Centre for Biomedical Science, St. Michael's Hospital, Toronto, Ontario, Canada; ${ }^{3}$ Institute of Physiology, Charité Universitätsmedizin Berlin, Berlin, Germany; ${ }^{4}$ Department of Medicine, University of Toronto, Toronto, Ontario, Canada; ${ }^{5}$ Institute for Vascular Signaling, Center for Molecular Medicine, Goethe University Frankfurt, Frankfurt, Germany; ${ }^{6}$ Department of Infectious Diseases and Pulmonary Medicine, Charité-Universitätsmedizin Berlin, Berlin, Germany; ${ }^{7}$ Department of Immunology, Max Planck Institute for Infection Biology, Berlin, Germany; ${ }^{8}$ Department of Surgery, University of Toronto, Toronto, Ontario, Canada; ${ }^{9}$ Department of Medicine/Division of Neurology, Duke Clinics for Pain and Palliative Care, Duke University Medical Center, Durham, North Carolina; and ${ }^{10}$ German Heart Institute Berlin, Berlin, Germany

\begin{abstract}
The cation channel transient receptor potential vanilloid (TRPV) 4 is expressed in endothelial and immune cells; however, its role in acute lung injury (ALI) is unclear. The functional relevance of TRPV4 was assessed in vivo, in isolated murine lungs, and in isolated neutrophils. Genetic deficiency of TRPV4 attenuated the functional, histological, and inflammatory hallmarks of acid-induced ALI. Similar protection was obtained with prophylactic administration of the TRPV4 inhibitor, GSK2193874; however, therapeutic administration of the TRPV4 inhibitor, HC-067047, after ALI induction had no beneficial effect. In isolated lungs, plateletactivating factor (PAF) increased vascular permeability in lungs perfused with $\operatorname{trp} v 4^{+/+}$more than with $\operatorname{trp} v 4^{-/-}$blood, independent of lung genotype, suggesting a contribution of TRPV4 on blood cells to lung vascular barrier failure. In neutrophils, TRPV4 inhibition or deficiency attenuated the PAF-induced increase in intracellular
\end{abstract}

calcium. PAF induced formation of epoxyeicosatrienoic acids by neutrophils, which, in turn, stimulated TRPV4-dependent $\mathrm{Ca}^{2+}$ signaling, whereas inhibition of epoxyeicosatrienoic acid formation inhibited the $\mathrm{Ca}^{2+}$ response to PAF. TRPV4 deficiency prevented neutrophil responses to proinflammatory stimuli, including the formation of reactive oxygen species, neutrophil adhesion, and chemotaxis, putatively due to reduced activation of Rac. In chimeric mice, however, the majority of protective effects in acid-induced ALI were attributable to genetic deficiency of TRPV4 in parenchymal tissue, whereas TRPV4 deficiency in circulating blood cells primarily reduced lung myeloperoxidase activity. Our findings identify TRPV4 as novel regulator of neutrophil activation and suggest contributions of both parenchymal and neutrophilic TRPV4 in the pathophysiology of ALI.

Keywords: transient receptor potential vanilloid 4; neutrophil; acute lung injury; calcium; reactive oxygen sepsis

(Received in original form June 6, 2014; accepted in final form July 26, 2015)

*These authors contributed equally to this work.

This work was supported by National Natural Science Foundation of China grants 81000028, 81370001, and 81300037; by the Natural Science Foundation of Jiangsu Province, China grant BK2010333; by the "333" Elitist Training Program, Jiangsu, China grant BRA2013135; by the "Six Talent Peaks" Training Program, Jiangsu, China grant WSN-078; by the Comprehensive Research Experience for Medical Students Program of the University of Toronto, Canadian Institutes of Health Research operating grants 97774 and 123417; by the Deutsche Forschungsgemeinschaft grants KU 1218/4-2 and Exzellenzcluster 147 "Cardio-Pulmonary Systems"; and by the Kaiserin-Friedrich Foundation, Berlin, Germany. The specific transient receptor potential vanilloid 4 inhibitor, GSK2193874, was kindly provided by GlaxoSmithKline LLC, Research Triangle Park, North Carolina.

Author Contributions: Conception and study design-J.Y. and W.M.K.; data acquisition, analysis, and interpretation—all authors; drafting the manuscriptJ.Y., C.T., and W.M.K.

Correspondence and requests for reprints should be addressed to Wolfgang M. Kuebler, M.D., Dr.Med., Keenan Research Centre for Biomedical Science, St. Michael's Hospital, 209 Victoria Street, Toronto, ON, M5B 1W8 Canada. E-mail: kueblerw@smh.ca

This article has an online supplement, which is accessible from this issue's table of contents at www.atsjournals.org

Am J Respir Cell Mol Biol Vol 54, Iss 3, pp 370-383, Mar 2016

Copyright @ 2016 by the American Thoracic Society

Originally Published in Press as DOI: 10.1165/rcmb.2014-02250C on July 29, 2015

Internet address: www.atsjournals.org 


\section{Clinical Relevance}

The present findings identify transient receptor potential vanilloid (TRPV) 4 as a novel regulator of neutrophil activation. In addition to the principal role of parenchymal TRPV4, neutrophilic TRPV4 contributes to features of experimental acute lung injury. This contribution may be of particular relevance in scenarios of systemic inflammation and direct neutrophil activation.

Acute respiratory distress syndrome (ARDS) is defined by acute hypoxemic respiratory failure, radiographic evidence of bilateral pulmonary opacities, and pulmonary edema, not fully explained by cardiac failure or fluid overload (1). Although no longer used to refer to the overarching clinical entity in humans, the term, "acute lung injury" (ALI) remains the proper descriptor of the corresponding disease in experimental animals. With an estimated incidence of 86.2 per 100,000 person-years and mortality rates of up to approximately $43 \%$ (2), ARDS presents a major cause of mortality and morbidity in critical care. Although extensive effort has been made to develop effective therapeutic strategies, including the introduction of recruitment maneuvers, high positive end-expiratory pressure application, high-frequency oscillatory ventilation, inhaled nitric oxide, or glucocorticoid administration (reviewed in Ref. 3), the only interventions demonstrated to improve clinical outcomes in ARDS so far are the use of a protective ventilatory strategy with low tidal volumes (4) and early application of prolonged prone positioning in patients with severe ARDS (5). In contrast, none of the pharmacological interventions tested so far in clinical trials could yield a detectable benefit in terms of mortality (3).

Although numerous direct (lungspecific; e.g., pneumonia) or indirect (systemic; e.g., sepsis) inflammatory triggers can cause ALI/ARDS, the resulting pathology and pathophysiology is strikingly uniform. The characteristic hallmarks of ALI/ARDS comprise diffuse endothelial and epithelial injury, which results in lung barrier failure and formation of a proteinaceous alveolar edema, and a strong inflammatory response characterized by the release of proinflammatory cytokines and the recruitment of granulocytes, monocytes, and platelets into the lung (6).

The transient receptor potential vanilloid (TRPV) 4 channel is one of six members of the vanilloid subfamily of transient receptor potential (TRP) channels, a group of 28 polymodal cation channels with multiple sensory functions that share some structural similarity (7). TRPV4 is widely expressed in lung parenchymal and inflammatory cells involved in ALI/ARDS, including lung microvascular endothelial cells (8), lung epithelial cells (8), neutrophils (9), and macrophages (10). TRPV4 channels are 5-10 times more permeable for $\mathrm{Ca}^{2+}$ than for $\mathrm{Na}^{+}$, and can be activated by a wide range of both physical stimuli, including cell swelling, heat, and mechanical stimulation. as well as chemical stimuli, such as endocannabinoids and epoxyeicosatrienoic acids derived from arachidonic acid (11).

Recently, we and others have shown TRPV4 to mediate the endothelial permeability increase and subsequent lung edema formation in response to increased hydrostatic stress $(12,13)$ and overventilation (10). Given the key role of endothelial permeability in ALI/ARDS and the wide range of TRPV4 activators, we considered that TRPV4-mediated vascular barrier failure may equally contribute to the pathophysiology of ALI. In addition, we speculated that TRPV4 may also modulate the inflammatory response in ALI, because: (1) TRPV4 is highly expressed in human leukocytes (14) and murine neutrophils (9); (2) TRPV4 has been implicated in the proinflammatory response of alveolar macrophages (10); and (3) the closely related channel TRPV2 was recently shown to play an essential role in phagocytes (15). It was therefore the objective of the present study to investigate the effect of genetic deficiency or pharmacological inhibition of TRPV4 on experimental ALI, and to identify its mechanistic role in the underlying inflammatory and permeability responses.

\section{Materials and Methods}

Details are provided in the accompanying online supplement. In brief, ALI was induced by intratracheal instillation of hydrochloric acid in male TRPV4-deficient $\left(\operatorname{trp} v 4^{-/-}\right)$mice (kindly provided by W. Liedtke, Duke University), their corresponding wild types $\left(\operatorname{trp} v 4^{+/+}\right)$, or chimeric mice generated by bone marrow transplantation between $\operatorname{trp} v 4^{+/+}$and $\operatorname{trp} v 4^{-/-}$mice after lethal irradiation. For prophylactic inhibition of TRPV4 in vivo, the specific inhibitor, GSK2193874, generously provided by GlaxoSmithKline (Research Triangle Park, NC), was injected intravenously 20 minutes before acid instillation. For all other applications, the commercially available TRPV4 inhibitor, HC-067047, was applied. As therapeutic approach, HC-067047, which we have previously shown to effectively prevent TRPV4-dependent ventilator-induced lung injury in mice (16), was administered 45 minutes after acid instillation. ALI was evaluated by measurements of quasistatic respiratory compliance, arterial oxygenation, lung edema, neutrophil influx, and histological signs of lung injury. In bronchoalveolar lavage (BAL) fluid, total protein content and inflammatory cytokine profiles were determined. In isolated, perfused mouse lungs, vascular permeability was assessed by Evan's blue extravasation and continuous monitoring of lung weight change in the presence of platelet-activating factor (PAF) or vehicle. Ex vivo chimeras were generated by perfusion of isolated lungs with heparinized whole blood, including platelets and neutrophils from donor mice $\left(\operatorname{trp} v 4^{+/+}\right.$or $\left.\operatorname{trp} v 4^{-1-}\right)$. Murine and human neutrophils were isolated as described previously (17, 18 ), and TRPV4 protein expression was determined by Western blot. The intracellular calcium concentration $\left(\left[\mathrm{Ca}^{2+}\right]_{\mathrm{i}}\right)$ in isolated neutrophils was measured by fura- 2 ratiometric imaging in the absence or presence of the TRPV4 inhibitor, HC-067047, before and after stimulation by PAF. Generation of reactive oxygen species (ROS) was quantified by luminol-enhanced chemiluminescence, neutrophil adhesion to lung microvascular endothelial cells pretreated with LPS was assessed, and neutrophil chemotaxis was determined by transwell assays. Rac activity in isolated neutrophils stimulated with PAF or vehicle (saline) was determined by an affinity precipitation assay (19) and epoxyeicosatrienoic acid (EET) profiles were quantified in cell supernatants, as previously described (20). Statistical analysis was performed by use of GraphPad Prism software (GraphPad Software Inc., 
La Jolla, CA). Data are presented as mean ( \pm SEM). Statistical analyses within groups were performed by Wilcoxon matched pairs signed rank test and repeated measures ANOVA on ranks (Friedman test and appropriate post hoc test). Different groups were compared by Mann-Whitney $U$ test and ANOVA on ranks (KruskalWallis test). Statistical significance was assumed at $P$ less than 0.05 .

\section{Results}

\section{TRPV4 in ALI}

In wild-type $\left(\operatorname{trp} v 4^{+/+}\right)$mice, instillation of hydrochloric acid caused characteristic functional features of murine ALI, including progressive arterial deoxygenation (Figure 1A), hypoxemia (Figure 1B), and reduced lung compliance (Figure 1C) after 2 hours. Lung vascular barrier failure was evident as increase in lung wet-to-dry weight ratio (Figure 1D) and BAL protein concentration (Figure 1E). Hallmarks of the lung inflammatory response were apparent as increased lung myeloperoxidase (MPO) activity, indicating influx of inflammatory neutrophils (Figure 1F) and elevated levels of the proinflammatory cytokines, IL-1 $\beta$, IL-6, monocyte chemotactic protein-1, keratinocyte-derived chemokine (KC), regulated upon activation, normal $\mathrm{T}$ cell expressed and secreted (RANTES), and macrophage inflammatory protein-2 (Figure 2). Lung injury was histologically evident in hematoxylin and eosin-stained lung sections, and quantitatively verified in blinded analyses by a lung injury score (Figures 2G and 2H).

Inhibition of TPRV4 in wild-type mice by prophylactic intravenous infusion of the TRPV4 inhibitor, GSK2193874 (5 mg/kg), 20 minutes before acid instillation significantly attenuated all features of acidinduced ALI (i.e., improved gas exchange and lung mechanics), and reduced signs of lung vascular barrier function, lung inflammation, and histological signs of lung injury (Figures 1 and 2). The key role of TRPV4 in experimental ALI was confirmed in a genetic loss-of-function model using TRPV4-deficient $\left(\operatorname{trp} v 4^{-/-}\right)$mice in which signs of ALI after acid instillation were again largely attenuated (Figures 1 and 2). Declaredly, we abstained from additional controls for pharmacological inhibition of genetic deficiency of TRPV4 in uninjured control mice, as these have recently been reported to show normal baseline characteristics of lung function, inflammation, and histology (21). Next, we tested the effects of TRPV4 inhibition in a clinically more relevant scenario, in that the TRPV4 inhibitor HC-067047 (1.6 mg/kg body weight) was administered 45 minutes after induction of ALI by acid instillation. At this time point, therapeutic TRPV4 inhibition no longer attenuated ALI, as assessed by parameters of impaired oxygenation (Figure 3A), respiratory mechanics (Figure 3B), lung barrier function (Figures 3C and 3D), neutrophil influx (Figure 3E), and histological evidence of lung injury (Figure 3F).

\section{Lung Vascular Barrier Regulation by TRPV4}

Based on our previously reported finding that TRPV4 is critically involved in the regulation of lung endothelial permeability, we tested for the role of TRPV4 in pulmonary vascular barrier function in isolated, buffer-perfused mouse lungs. Stimulation with PAF ( 5 nmol bolus, final concentration in perfusate: $1 \mu \mathrm{mol} / \mathrm{L}$ ) significantly increased lung weight gain (Figure 4A) and Evans blue extravasation (Figure 4B) in isolated lungs from wild-type mice, indicating relevant protein and fluid leakage across the endothelium that was largely attenuated in lungs from $\operatorname{trp} v 4^{-1-}$ mice. Conversely, to address the contribution of TRPV4 expression on circulating blood cells, we performed chimeric cross-transfusion experiments in isolated blood-perfused lungs. Notably, whereas PAF stimulation in the bufferperfused lung is a well established model of direct PAF-induced endothelial leak (22), PAF infusion into the blood-perfused lung presents primarily a model of PAF activation of circulating blood cells, as PAF will be rapidly deactivated by plasma PAF acetylhydrolase (23) before reaching the alveolar capillary bed. In $\operatorname{trp} v 4^{+/+}$lungs perfused with $\operatorname{trp} v 4^{+/+}$blood, PAF stimulation increased Evans blue extravasation as compared with unstimulated blood-perfused lungs (Figure 4C). In contrast to the results in buffer-perfused lungs, PAF-induced vascular leak was not attenuated by TRPV4 deficiency in lung parenchymal cells, but significantly reduced in lungs perfused with blood from $\operatorname{trp} v 4^{-1-}$ mice independent of the genotype of the perfused lung.
Perfusion pressures in these experiments increased from $13.0( \pm 1.0)$ to $21.6( \pm 3.4)$ $\mathrm{cm} \mathrm{H}_{2} \mathrm{O}$ in response to PAF, yet did not differ between lung or blood genotypes, respectively, indicating that differences in Evans blue extravasation reflected changes in vascular permeability rather than hydrostatic pressure (data not shown). Taken together, these findings indicate that, depending on the stimulation mode, functional expression of TRPV4 on both parenchymal cells and circulating blood cells can contribute to lung vascular barrier failure.

\section{Role of TRPV4 in Neutrophil Function}

To our knowledge, TRPV4-dependent regulation of circulating blood cell functions has not been reported as of yet; however, previous studies have reported high expression levels of TRPV4 in human leukocytes (14) and murine neutrophils (9). Because neutrophils play a central role in the pathogenesis of ALI and lung vascular barrier failure, we speculated that TRPV4 may critically regulate neutrophil function. We first confirmed TRPV4 expression on isolated neutrophils from both human volunteers and mice (Figure 5A). Next, we tested whether TRPV4 is required for the $\left[\mathrm{Ca}^{2+}\right]_{\mathrm{i}}$ response of neutrophils to proinflammatory stimuli. In neutrophils of $\operatorname{trp} v 4^{+/+}$mice, addition of PAF induced a transient increase in $\left[\mathrm{Ca}^{2+}\right]_{\mathrm{i}}$ that showed the rapid upslope characteristic for the well documented, $\mathrm{IP}_{3}$-dependent $\mathrm{Ca}^{2+}$ release from intracellular stores, and the subsequent prolonged elevation attributable to influx of extracellular $\mathrm{Ca}^{2+}(24)$. The latter component of the $\left[\mathrm{Ca}^{2+}\right]_{\mathrm{i}}$ response was markedly reduced in neutrophils of $\operatorname{trp} v 4^{-1-}$ mice, or in the presence of the TRPV4 inhibitor, HC-067047 (25) (Figures $5 \mathrm{~B}$ and $5 \mathrm{C}$ ), demonstrating that TRPV4 was required for a sustained $\left[\mathrm{Ca}^{2+}\right]_{\mathrm{i}}$ elevation in response to PAF. Similarly to the effects in murine cells, HC-067047 attenuated the $\left[\mathrm{Ca}^{2+}\right]_{\mathrm{i}}$ response to PAF in human neutrophils (Figure 5D).

EETs are potent activators of TRPV4 in vascular endothelial cells $(11,26)$. Analogously, stimulation with $3 \mu \mathrm{mol} / \mathrm{L}$ 5,6-EET, a concentration previously shown to activate endothelial TRPV4 (8), increased $\left[\mathrm{Ca}^{2+}\right]_{\mathrm{i}}$ in murine neutrophils of wild-type mice, but significantly less so in neutrophils of $\operatorname{trp} v 4^{-l-}$ mice (Figure $5 \mathrm{E}$ ). Stimulation with PAF triggered the formation of several EETs, including 5,6EET, 8,9-EET, 11,12-EET, and 14,15-EET, 
A

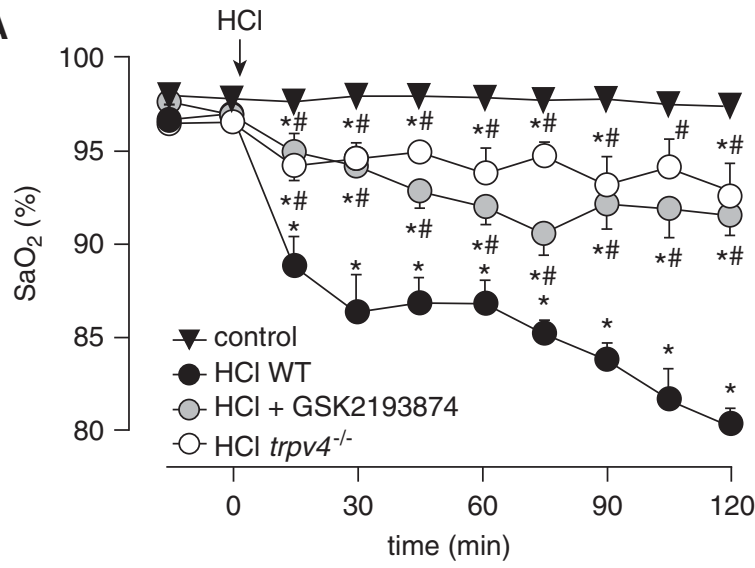

C

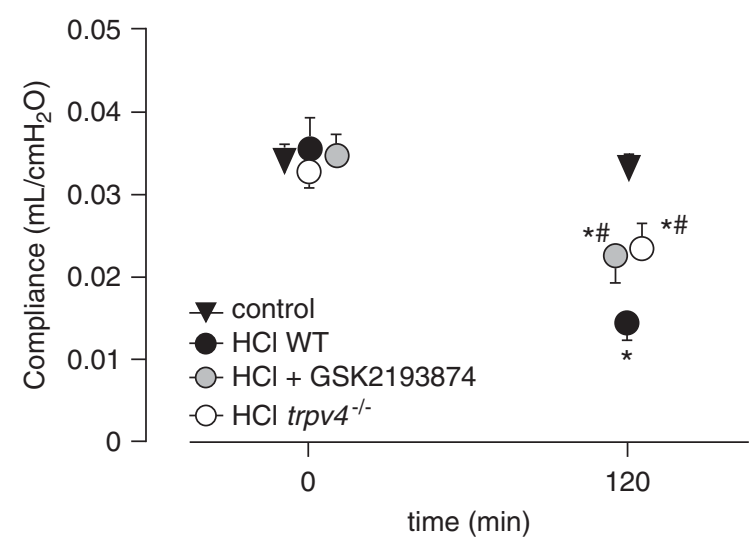

E

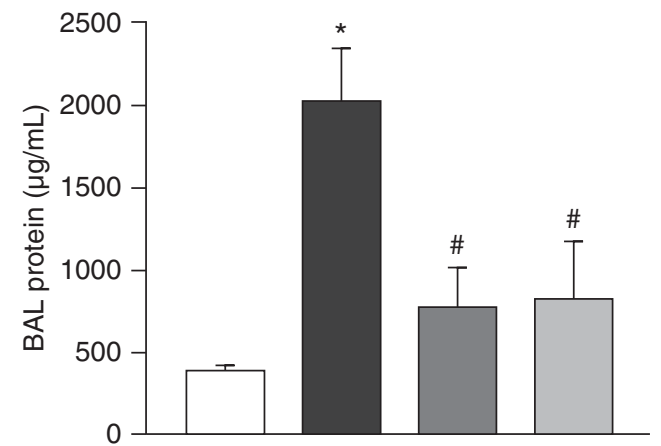

B

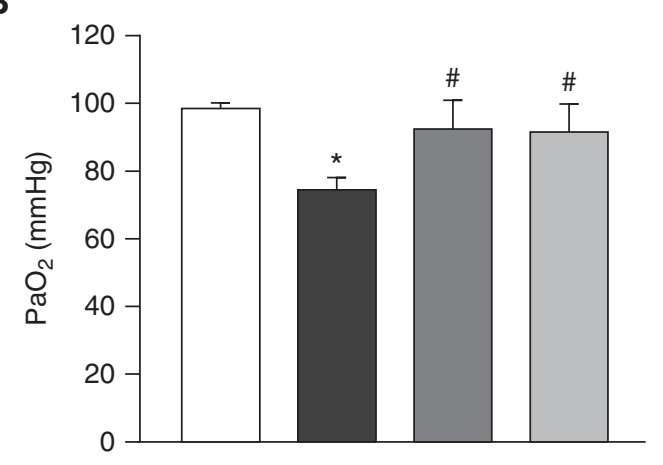

D

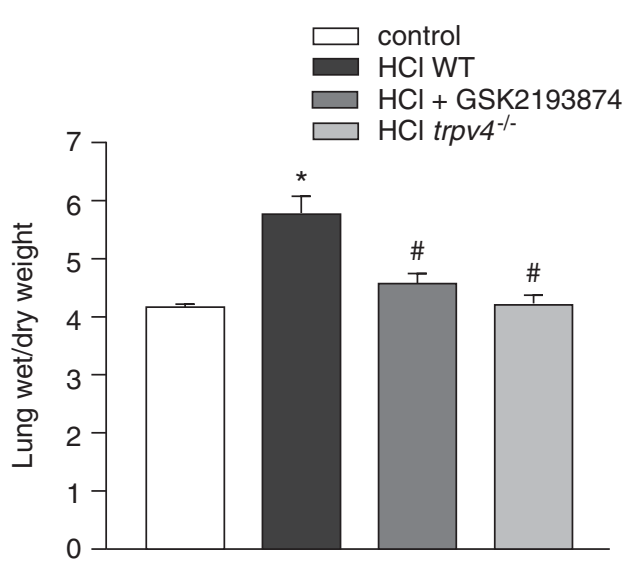

$\mathbf{F}$

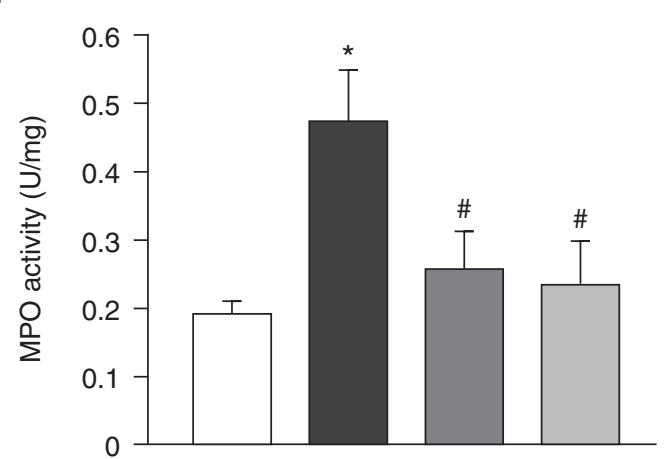

Figure 1. Prophylactic transient receptor potential vanilloid (TRPV) 4 inhibition or deficiency attenuate physiological characteristics of acute lung injury (ALI). Group data show the effects of pharmacological TRPV4 inhibition by GSK2193874 (5 mg/kg, delivered 20 minutes before the induction of lung injury) or genetic deficiency of TRPV4 (trpv4 ${ }^{-/-}$) in a 2-hour murine model of acid (HCl)-induced ALI on arterial oxygen saturation (Sa $\mathrm{O}_{2}$; $A$ ) and partial arterial oxygen pressure $\left(\mathrm{Pa}_{\mathrm{O}_{2}} ; B\right)$, lung quasistatic compliance $(C)$, lung wet-to-dry-weight ratio $(D)$, protein concentration in the bronchoalveolar lavage (BAL) fluid (E), and lung myeloperoxidase (MPO) activity as a measure of neutrophil invasion (F). Data are mean \pm SEM from $n=9,9,5,9,9$, and 7 mice per group in $A-F$, respectively. ${ }^{*} P<0.05$ versus healthy control mice; ${ }^{*} P<0.05$ versus $\mathrm{HCl}$ in untreated wild-type (WT) mice.

in human neutrophils (Figure 5F), and cytochrome P450 epoxygenase inhibition using propargyloxyphenyl hexanoic acid $(50 \mu \mathrm{Mol} / \mathrm{L})(12)$ attenuated the neutrophil $\left[\mathrm{Ca}^{2+}\right]_{\mathrm{i}}$ response to PAF (Figure $5 \mathrm{G}$ ).

Taken together, these data suggest that PAF triggers a $\left[\mathrm{Ca}^{2+}\right]_{\mathrm{i}}$ increase in neutrophils via formation of EETs, which, in turn, stimulate TRPV4 cation channels, allowing for sustained $\mathrm{Ca}^{2+}$ influx.

Next, we tested for functional downstream effects of TRPV4 activation in neutrophils. In wild-type murine cells, stimulation with PAF caused a rapid, pronounced, and sustained production of ROS characteristic of the respiratory burst of neutrophils (Figure 6A). In neutrophils of $\operatorname{trp} v 4^{-1-}$ mice, however, this response 


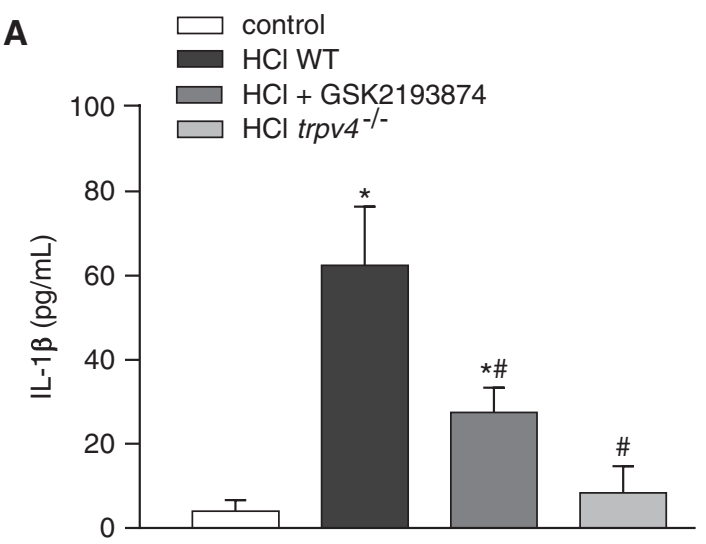

C

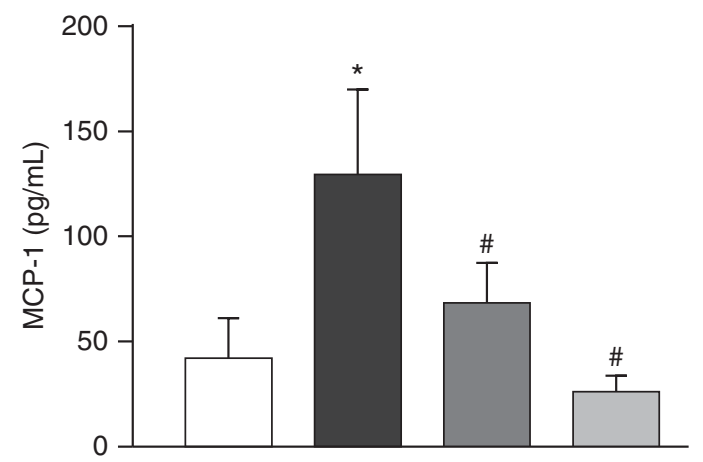

$\mathbf{E}$

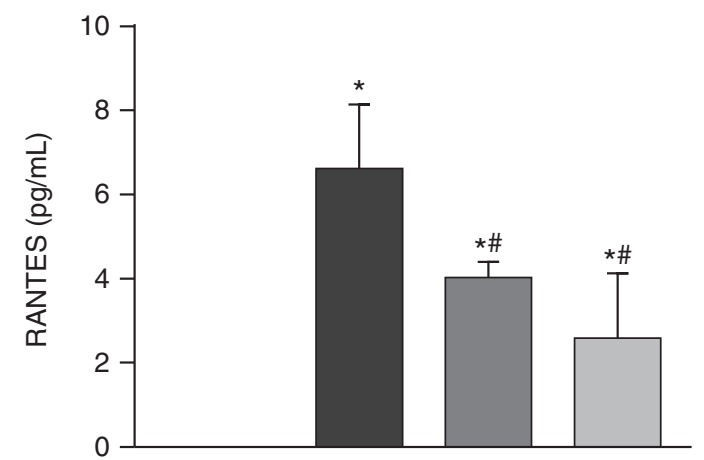

B

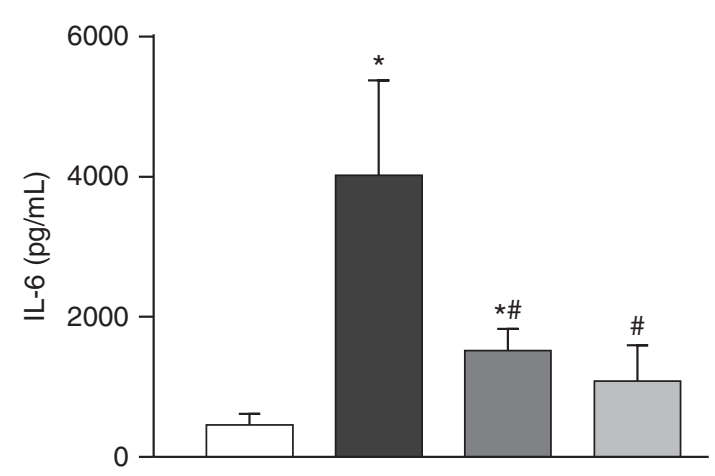

D

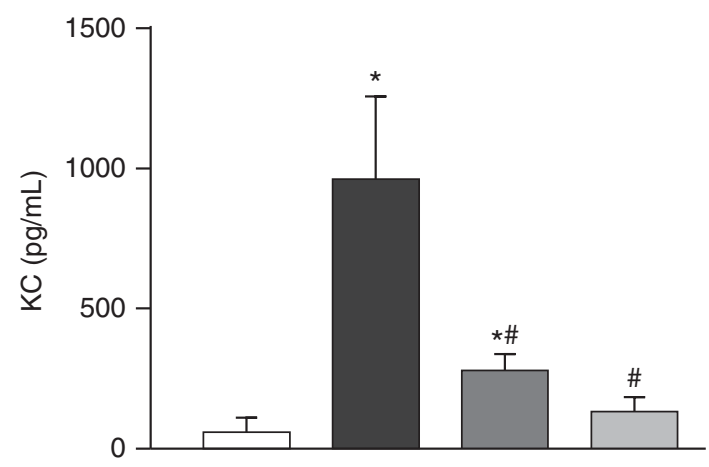

$\mathbf{F}$

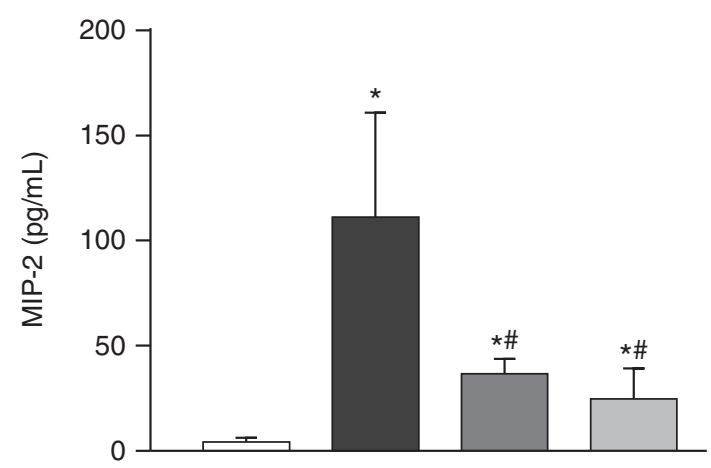

Figure 2. Prophylactic TRPV4 inhibition or deficiency attenuate the lung inflammatory cytokine response and histological evidence of ALI. Group data show the effects of pharmacological TRPV4 inhibition by GSK2193874 (5 mg/kg, delivered 20 minutes before the induction of lung injury) or genetic deficiency of TRPV4 (trpv4 ${ }^{-1-}$ ) in a 2-hour murine model of acid $(\mathrm{HCl})$-induced ALI on the concentration of IL-1 $\beta(A)$, IL-6 (B), monocyte chemotactic protein (MCP)-1 $(C)$, keratinocyte-derived chemokine $(K C)(D)$, regulated upon activation, normal T cell expressed and secreted (RANTES) (E), and macrophage inflammatory protein (MIP)-2 $(F)$ in the BAL fluid. Histological features of lung injury are shown by representative histological micrographs $(G)$ and as group data on a quantitative scale from 0 (no injury) to 1 (maximal) $(H)$. Data are mean \pm SEM from $n=6$ mice per group in $A-F, n=5$ mice in control, and $n=10$ mice in all other groups in $H .{ }^{*} P<0.05$ versus healthy control mice; ${ }^{\#} P<0.05$ versus $\mathrm{HCl}$ in untreated WT mice.

was largely attenuated. Consistent with a key role of TRPV4 in neutrophil activation, neutrophil adhesion to LPS-stimulated lung microvascular endothelial cells (Figure 6B) and neutrophil transmigration across an endothelial monolayer (Figure 6C) were markedly reduced when neutrophils were deficient in functional
TRPV4 or pretreated with the TRPV4 inhibitor HC-067047. As activation of the small GTPase, Rac, constitutes a common denominator for the respiratory burst of neutrophils (27), leukocyte adhesion (28), and chemotaxis (29), and can be triggered by $\mathrm{Ca}^{2+}$ influx (30), we probed for the role of TRPV4 in neutrophil
Rac activation. In neutrophils of wild-type mice, stimulation with PAF approximately doubled the ratio of active versus total Rac, yet this effect was completely absent in neutrophils of $\operatorname{trp} v 4^{-1-}$ mice (Figures 6D and $6 \mathrm{E}$ ), indicating that PAF-induced Rac activation in neutrophils requires TRPV4. Of note, TRPV4 deficiency did 
G
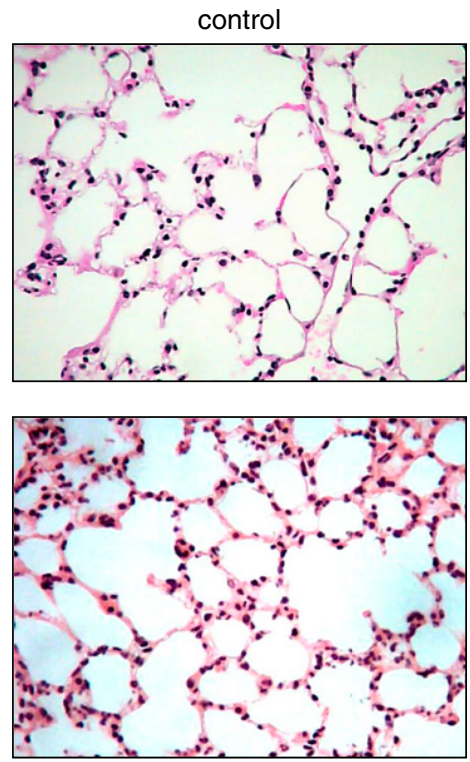

$\mathrm{HCl}+$ GSK2193874
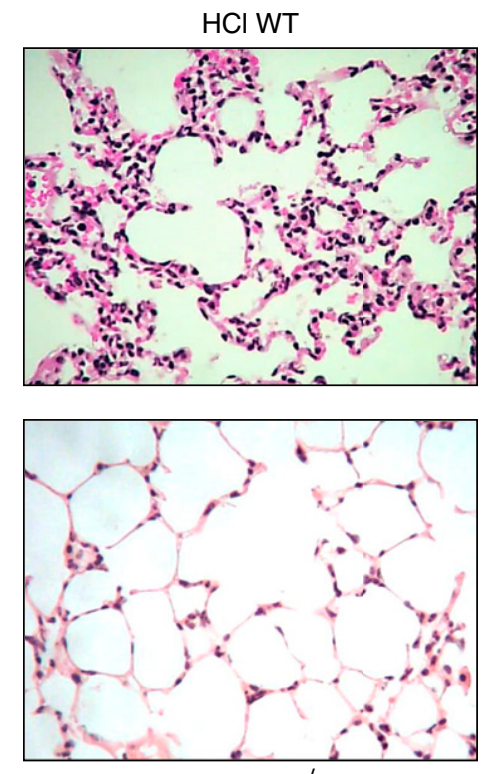

$\mathrm{HCl}$ trpv $4^{-/-}$
H

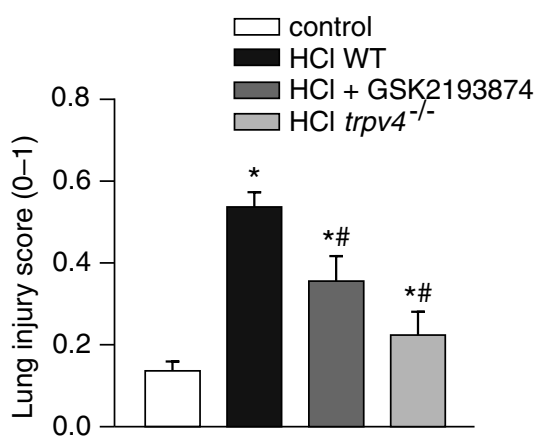

Figure 2. (Continued).

not affect total numbers of circulating white blood cells or neutrophils in mice (Figure 6F).

\section{Acid-Induced Lung Injury in TRPV4 Chimeric Mice}

Finally, to probe for the relative contribution of endothelial versus neutrophilic TRPV4 in our murine model of acid-induced lung injury, we generated TRPV 4 chimeric mice by bone marrow transfer from $\operatorname{trpv} 4^{+/+}$

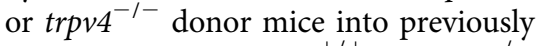
lethally irradiated $\operatorname{trpv4^{+/+}}$ or $\operatorname{trpv4^{-l-}}$ recipient mice, respectively. Controls without ALI did not differ between genotypes and chimeras in lung wet-to-dryweight ratio (4.51 \pm 0.05$)$, histological lung injury score $(0.084 \pm 0.016)$, BAL protein $(0.21 \pm 0.01 \mu \mathrm{g} / \mathrm{ml})$, and MPO activity $(0.21 \pm 0.05 \mathrm{U} / \mathrm{mg})$, respectively $(n=3$ per group each). Pulmonary vascular leakage in response to acid instillation, as measured by the lung wet-to-dry-weight ratio, was largely abrogated by genetic deficiency of TRPV4 in parenchymal tissue, but not by TRPV4 deficiency in circulating blood cells (Figure 7A). Similarly, histological lung injury scores did not reveal a beneficial effect of TRPV4 deficiency in circulating blood cells, whereas parenchymal TRPV4 deficiency showed similar protection as combined TRPV4 deficiency in both lung and blood cells (Figure 7B), and comparable protection as initially detected in $\operatorname{trpv4^{-1-}}$ mice (Figure 2H). Selective deficiency of TRPV4 in neither parenchymal nor myeloid cells conferred significant protection against protein leakage into the $\mathrm{BAL}$, whereas $\mathrm{BAL}$ protein content was significantly reduced in mice with complete TRPV4 deficiency (Figure 7C). In contrast, the increase in MPO activity was significantly reduced by TRPV4 deficiency in either lung parenchymal or circulating blood cells, indicating a role for both endothelial and neutrophilic TRPV4 in the accumulation of neutrophils in the acid-injured lung (Figure 7D).

\section{Discussion}

In the present work, we identify a critical role of the polymodal cation channel TRPV4 in the pathogenesis of ALI and in the activation of neutrophils. In a murine model of acid-induced lung injury, which mimics the clinical development of ARDS after aspiration of gastric contents (31), both genetic deficiency and prophylactic pharmacological inhibition of TRPV4 markedly attenuated the key features of experimental ALI (32) (i.e., impairment of gas exchange and lung function, inflammation, lung edema, and histological evidence of lung damage, respectively). The protective effect of TRPV4 inhibition was, however, lost when the inhibitor was administered therapeutically 45 minutes after ALI induction. Isolated, bufferperfused lungs from $\operatorname{trp} v 4^{-1-}$ mice were protected from PAF-induced barrier failure, suggesting involvement of TRPV4 expressed on lung parenchymal cells; however, chimeric experiments in bloodperfused lungs revealed, in addition, a critical role of TRPV4 expression on circulating blood cells. We confirmed expression of TRPV4 on both murine and human neutrophils, and demonstrated its functional relevance for mediating the sustained neutrophil $\mathrm{Ca}^{2+}$ response to PAF. The latter was likely triggered by EETs, as the PAF-induced increase in neutrophil $\left[\mathrm{Ca}^{2+}\right]_{\mathrm{i}}$ was sensitive to a cytochrome $\mathrm{P} 450$ epoxygenase inhibitor. This view was substantiated by the findings that PAF triggered the formation of various bioactive EETs in neutrophils, and that exogenous 5,6-EET in turn stimulated neutrophil $\mathrm{Ca}^{2+}$ signaling in a TRPV4sensitive manner. Furthermore, we found TRPV4 to be critical for neutrophil activation and function in response to PAF, as respiratory burst, neutrophil adhesion, and migration were markedly reduced in trpv $4^{-1-}$ cells, a fact that may be partially attributable to a lesser activation of the small GTPase Rac. Bone marrow chimeras showed, however, that the pathogenic relevance of TRPV4 in murine, acidinduced lung injury was primarily related 
A

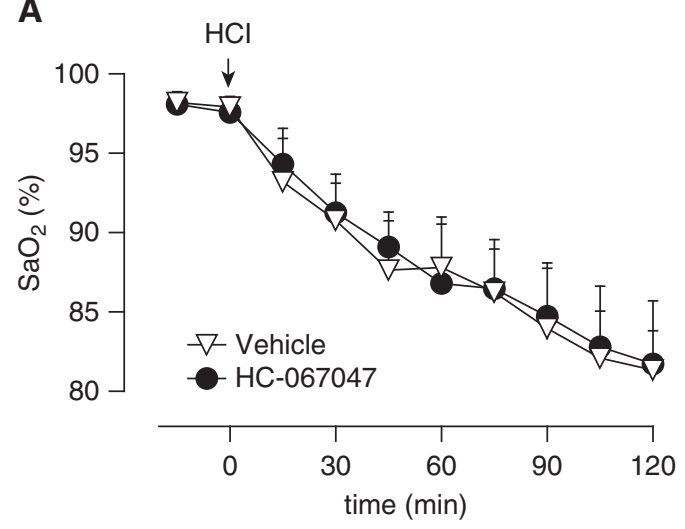

C

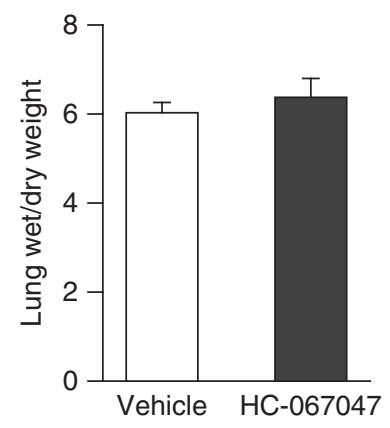

E

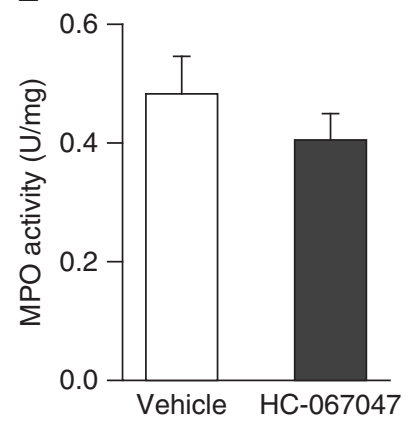

B

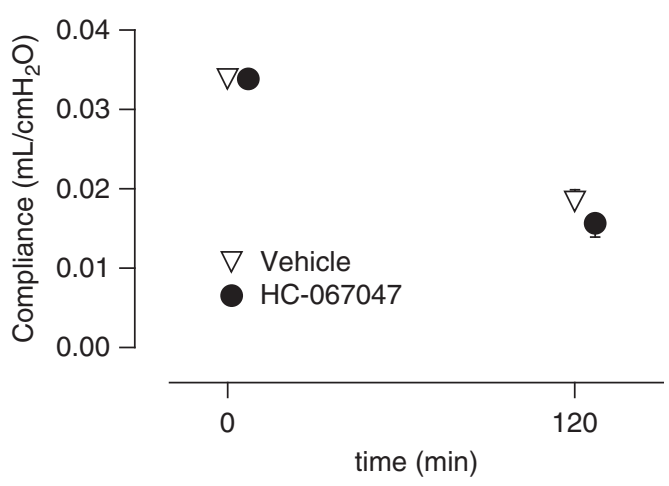

D

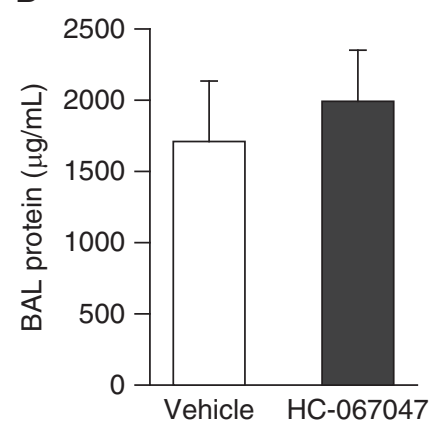

$\mathbf{F}$

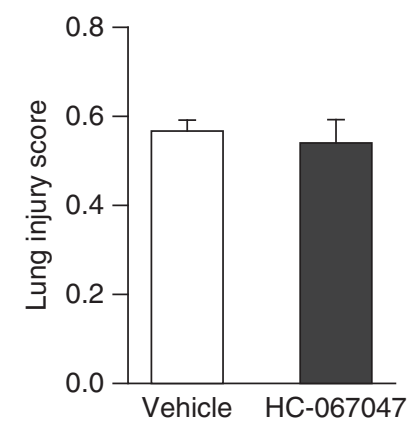

Figure 3. Therapeutic TRPV4 inhibition does not alleviate characteristic features of acid-induced lung injury. Group data show the effects of pharmacological TRPV4 inhibition by HC-067047 (1.6 mg/kg, delivered 45 minutes after the induction of lung injury) in a 2-hour murine model of acid $(\mathrm{HCl})$-induced $\mathrm{ALI}$ on $\mathrm{Sa}_{2}(A)$, lung quasi-static compliance $(B)$, lung wet-to-dry weight ratio $(C)$, protein concentration in the BAL fluid $(D)$; lung MPO activity as a measure of neutrophil invasion $(E)$, and histological features of lung injury on a quantitative scale from 0 (no injury) to 1 (maximal) $(F)$. Data are mean \pm SEM from $n=10$ mice in control, and $n=8$ mice in HC-067047 for $A-F$; no significant differences were detected between groups.

to endothelial, not neutrophilic, TRPV4, with the latter only contributing to neutrophil accumulation, but not relevantly to vascular leak or histological signs of lung injury.

Taken together, our findings reveal a previously unrecognized role of TRPV4 in neutrophil activation and function that can contribute to some features of ALI, yet the predominant relevance of TRPV4 in ALI relates to its expression in lung parenchymal cells.
In the present study, we probed for the role of TRPV4 in experimental ALI, lung vascular leak and neutrophil activation using the well established in vivo model of acid-induced lung injury on the one hand (31), and PAF as stimulus in our in situ and in vitro experiments on the other hand. The latter choice was based on the following considerations: (1) that PAF has been implicated as an important mediator in clinical ARDS, and its blockage is protective in most experimental models of ALI (33); and (2) that PAF can activate neutrophils (24) and is concomitantly one of the few mediators that will directly and rapidly induce endothelial barrier dysfunction in the intact lung (34), which makes it ideally suited to address the individual contributions of both endothelial and neutrophilic TRPV4. However, it is important to keep in mind that in situ or in vitro stimulation with $\mathrm{PAF}$, like any other single mediator, only reflects a 

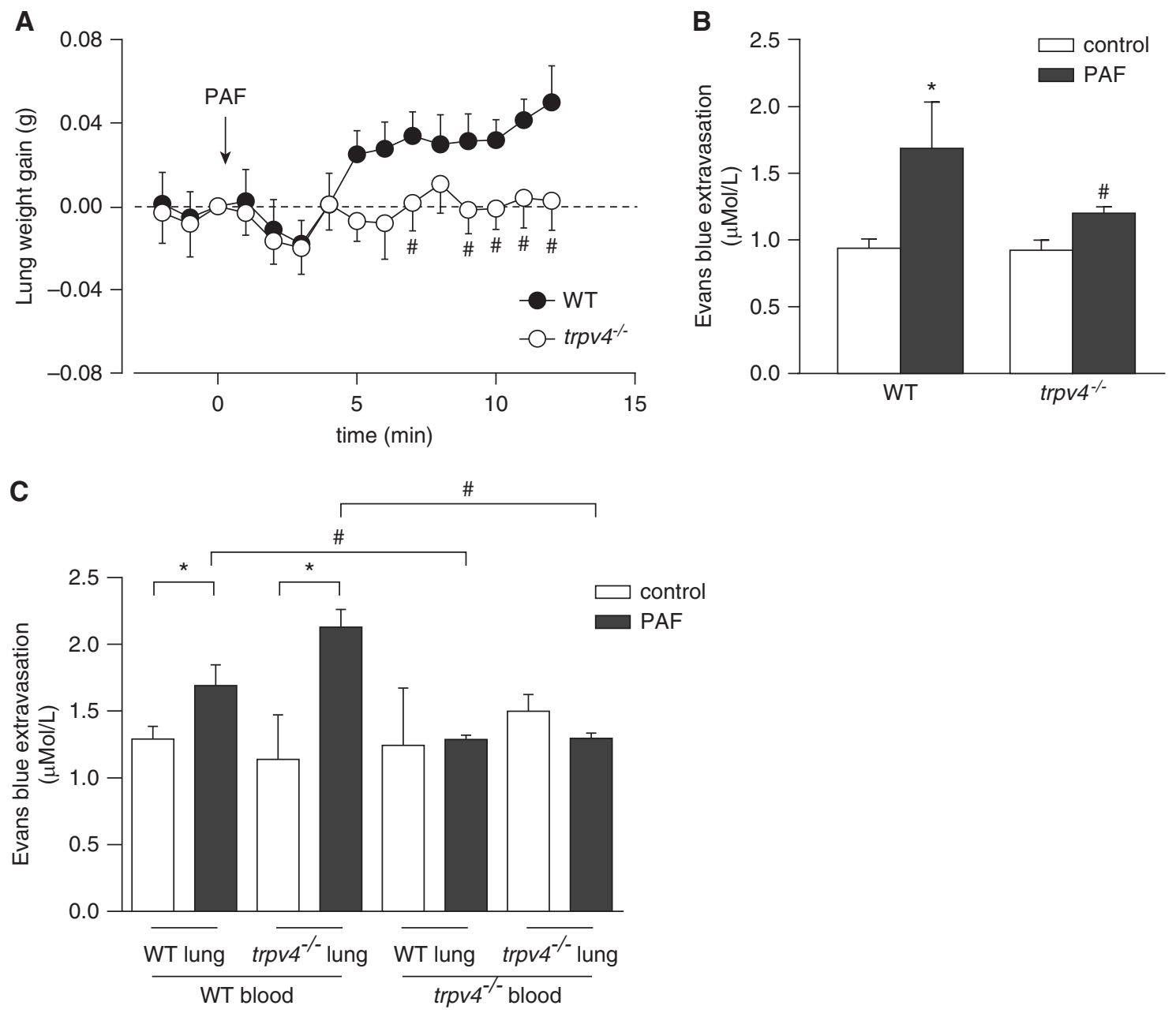

Figure 4. The role of TRPV4 in vascular barrier failure of blood-perfused lungs is partially attributable to its expression on circulating blood cells. Isolated, buffer-perfused lungs of TRPV4-deficient (trpv $4^{-1-}$ ) or corresponding WT mice were stimulated with a bolus of platelet-activating factor (PAF; 5 nmol; final concentration in perfusate: $1 \mu \mathrm{mol} / \mathrm{L}$ ) or vehicle (DMSO; control), and vascular barrier failure was assessed as either increase in total lung weight (A) or as extravasation of Evans blue $(B)$, respectively. For chimeric experiments, isolated lungs from either trpv $4^{-1-}$ or WT mice were perfused with heparinized whole blood from trpv $4^{-1-}$ or WT mice, respectively, and barrier failure in response to PAF bolus infusion (5 nmol) was determined as Evans blue extravasation (C). Data are mean \pm SEM from $n=5$ and 7 lungs per group in $A$ and $B$, and $n=3$ for controls and $n=7$ for PAF groups in $C$, respectively. ${ }^{\star} P<0.05$ versus control; ${ }^{\#} P<0.05$ versus corresponding WT lungs $(A$ and $B$ ) or between different blood genotypes as indicated $(C)$.

simplified model of the complex scenario of ALI in vivo. Furthermore, acid-induced lung injury in vivo and PAF stimulation in isolated lungs not only differ in the complexity of the two models, but also in their site of action, in that intratracheal acid instillation primarily causes epithelial injury, whereas addition of PAF to the lung perfusate will primarily target the vascular endothelium in bufferperfused lungs, or-due to the rapid degradation of PAF by plasma PAF acetylhydrolase (23) - the circulating blood cells in blood-perfused lungs, respectively.

TRPV4 is a widely expressed polymodal sensory cation channel that is critically involved in a broad variety of cell and organ functions, ranging from bone development to the regulation of vascular tone to renal regulation of urine output and tonicity (35). Human gene mutations in TRPV4 are associated with various forms of skeletal dysplasias and neurodegenerative diseases (36).

Importantly, TRPV4 is not only expressed on the key cell types involved in the pathogenesis of ALI (i.e., lung endothelial [8] and epithelial [8] cells, as well as alveolar macrophages [10] and neutrophils [9]), but also functionally regulates cellular responses that are considered characteristic hallmarks in the pathogenesis of ALI (37), such as lung endothelial barrier failure $(12,13)$ and macrophage activation (10). We therefore postulated that TRPV4 may contribute relevantly to ALI, and confirmed this hypothesis by demonstrating that genetic deficiency or pharmacological inhibition of TRPV4 largely attenuated the classic features of organ damage (i.e., loss of function, inflammation, and permeability edema) in a murine model of acid-induced ALI. Whereas the blocker potency of the applied TRPV4 inhibitor, GSK2193874, is lower by approximately a factor of 10 in human as compared with murine or rat TRPV4, its close analog, GSK2263095, has a comparable half maximal inhibitory concentration $\left(\mathrm{IC}_{50}\right)$ for human TRPV4 
A

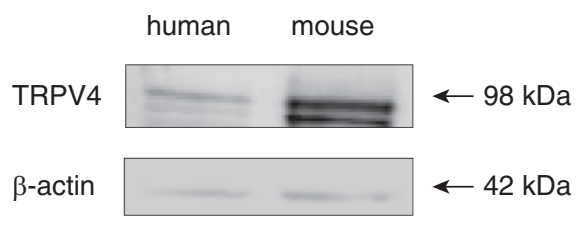

C

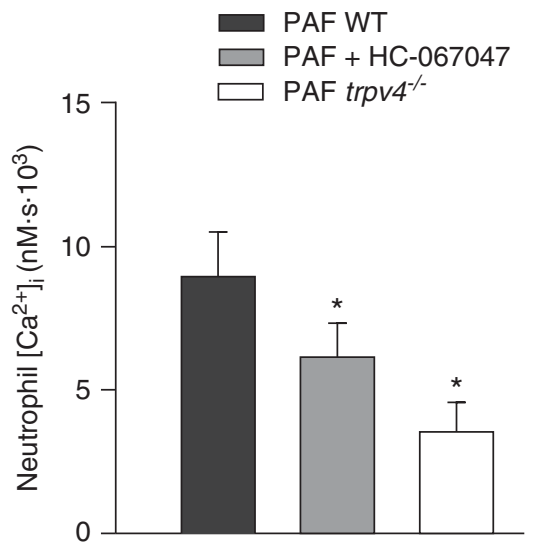

$\mathbf{F}$

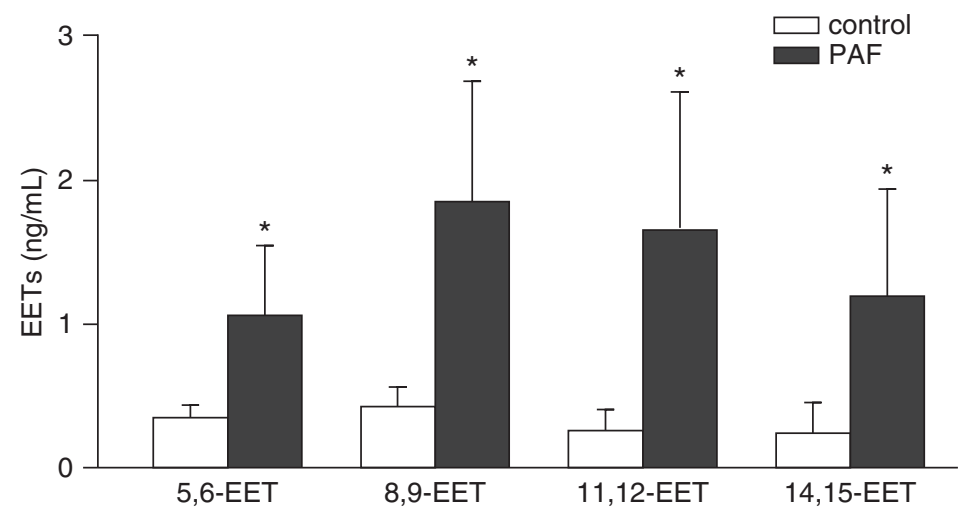

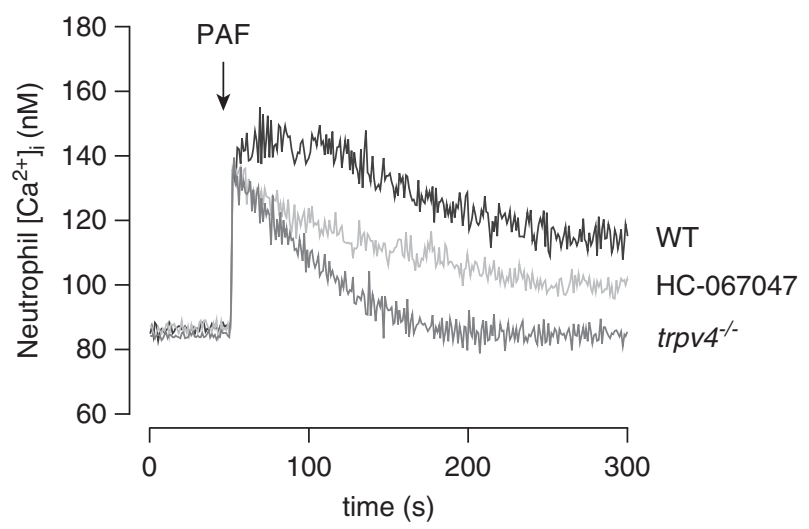

D

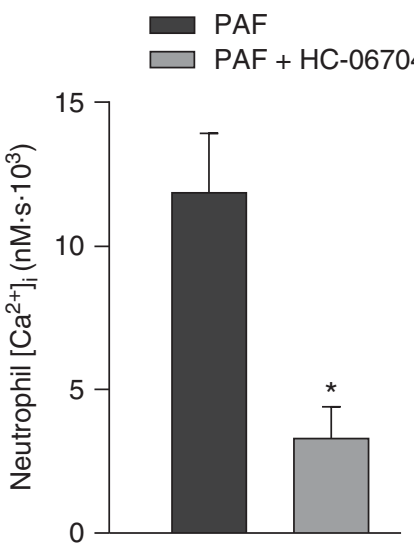

E

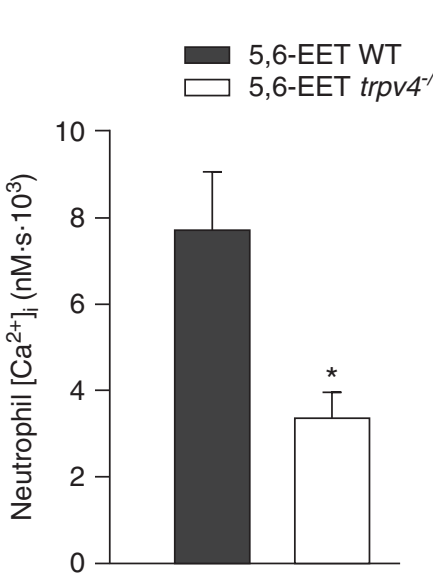

G

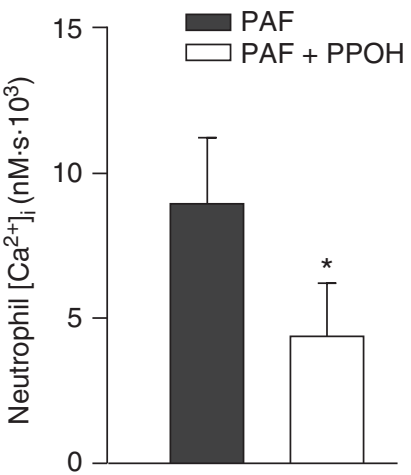

Figure 5. TRPV4 mediates the neutrophil calcium response to PAF. Western blot analyses show expression of TRPV4 in isolated neutrophils (A). Representative tracings $(B)$ and group data $\left.\left(C \text {; as area-under-the-curve over baseline for } 5 \text { minutes) show intracellular calcium concentration ([Ca }{ }^{2+}\right]_{i}\right)$ response to PAF $(10 \mu \mathrm{mol} / \mathrm{L})$ stimulation in murine neutrophils from WT mice in the absence or presence of the TRPV4 inhibitor, HC-067047 (20 $\mu \mathrm{mol} / \mathrm{L})$, or in neutrophils isolated from TRPV4-deficient (trpv $4^{-/-}$) mice, and in human neutrophils in the presence or absence of HC-067047 (D). Group data show $\left[\mathrm{Ca}^{2+}\right]_{i}$ response to 5,6-epoxyeicosatrienoic acid (EET; $\left.3 \mu \mathrm{mol} / \mathrm{L}\right)$ in murine neutrophils from WT and trpv $4^{-1-}$ mice $(E)$, and formation of the regioisomers 5,6-EET, 8,9-EET, 11,12-EET, and 14,15-EET in human neutrophils after 5-minute stimulation with PAF (10 $\mu$ mol/L; F). Group data show $\left[\mathrm{Ca}^{2+}\right]_{\mathrm{i}}$ response to PAF $(10 \mu \mathrm{mol} / \mathrm{L})$ in human neutrophils in the presence or absence of the cytochrome P450 epoxygenase inhibitor, propargyloxyphenylhexanoic acid $(\mathrm{PPOH} ; 50 \mu \mathrm{Mol} / L)(G)$. Data are mean \pm SEM from $n=6$ per group in $C-E$ and $G$, and $n=4$ per group in $F$. ${ }^{*} P<0.05$ versus WT PAF $(C)$, PAF ( $D$ and $G), 5,6-E E T W T(E)$, or control $(F)$, respectively.

(3 $\mathrm{nM}$ ), as GSK2193874 does for murine and rat TRPV4 (2-5 nM) after stimulation with the selective TRPV4 agonist,

GSK634775 (38). Although this renders the use of TRPV4 blockers for the prevention and/or treatment of clinical ARDS a realistic possibility, therapeutic administration of a TRPV4 inhibitor did not prove successful in attenuating ALI when administered 45 minutes after intratracheal acid instillation. This result is somewhat surprising, given that 
A

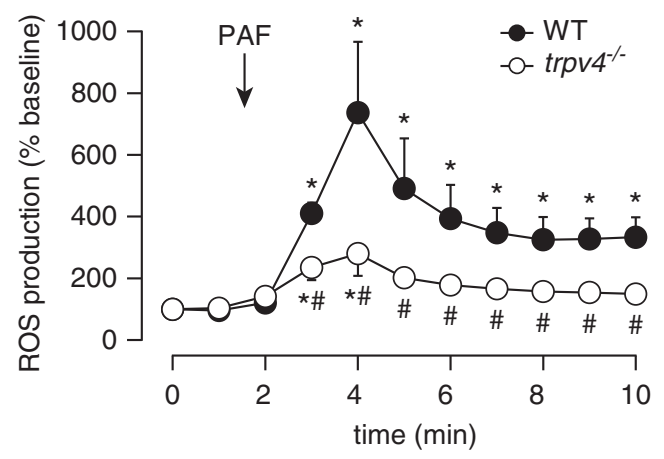

C

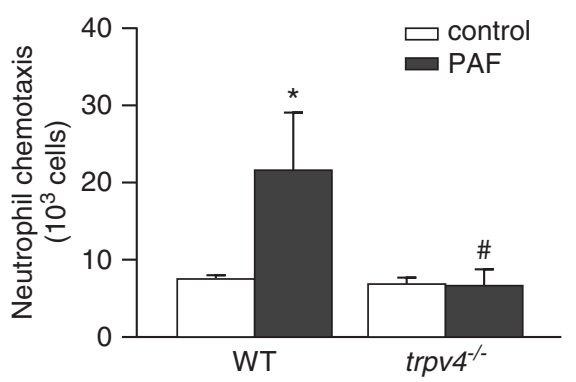

E

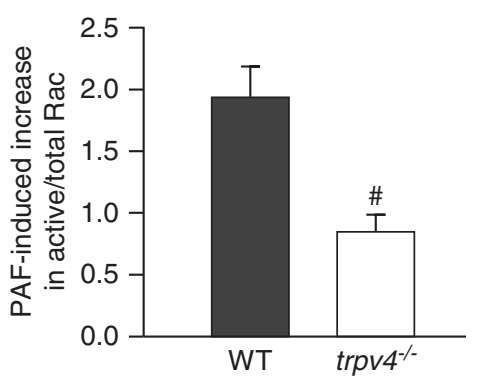

B

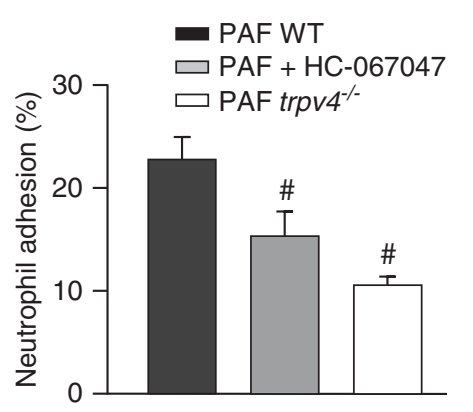

of murine ventilator-induced lung injury, where it conveyed protection similar to genetic TRPV4 deficiency when given at a similar dose and by similar route, as in the present study (16). Hence, it seems that therapeutic administration of TRPV4 inhibitors may alleviate lung injury in some, but not all, experimental models, and that further studies in different disease models and species are needed before translation of this approach to the clinical scenario.

Based on previous findings by us (13) and others (39), which have demonstrated an important role of TRPV4 in the regulation of lung vascular barrier integrity, we initially hypothesized that the protective effect of TRPV4 inhibition or deficiency in experimental ALI was predominantly attributable to its expression on lung microvascular endothelial cells. We tested this concept in isolated, perfused lungs by measuring the lung vascular barrier response to $\mathrm{PAF}$, one of the few mediators that can increase pulmonary vascular permeability within minutes in the absence of inflammatory blood cells (34). Initial experiments in isolated, buffer-perfused lungs confirmed this notion, insofar as lung weight gain and Evans blue extravasation in response to PAF were largely prevented in lungs of $\operatorname{trp} v 4^{-I-}$ mice. Notably, PAF-induced increases in lung perfusion pressure did not differ between genotypes, confirming that differences in fluid accumulation and leak reflected TRPV4-dependent changes in lung vascular permeability rather than hydrostatic pressure. Unexpectedly, however, subsequent chimeric experiments in blood-perfused, isolated lungs identified TRPV4 expression on circulating blood cells as additional putative mechanism of PAF-induced lung vascular barrier failure, in that PAFinduced Evans blue extravasation was largely blocked when lungs were perfused with TRPV4-deficient blood, independent of the actual lung genotype. Importantly, although the buffer-perfused lung is a well established model of direct PAFinduced endothelial leak, the bloodperfused lung reflects primarily a model of PAF-induced neutrophil activation and secondary endothelial leak. Hence, although experiments in buffer-perfused lungs substantiated the established role of endothelial TRPV4 in lung vascular barrier regulation, subsequent 
A

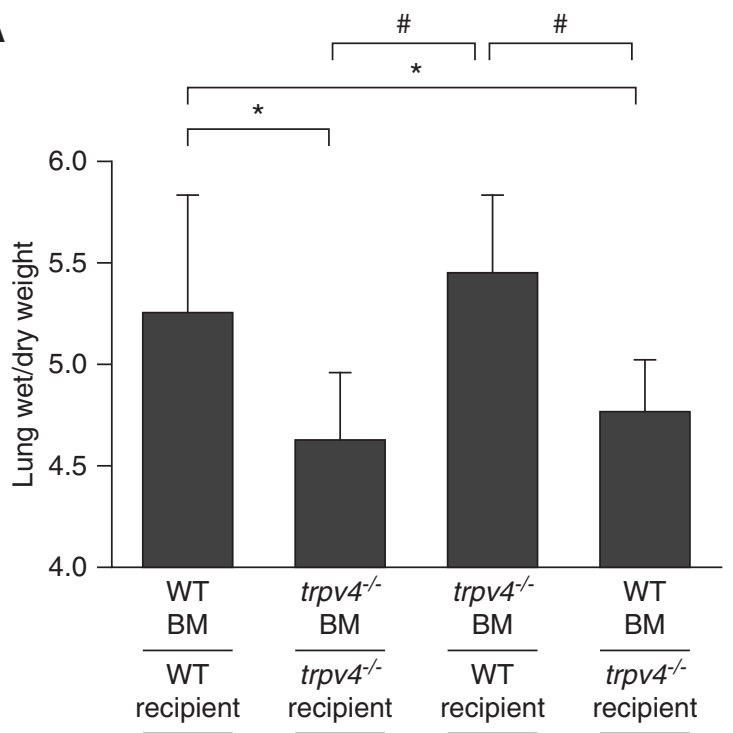

C

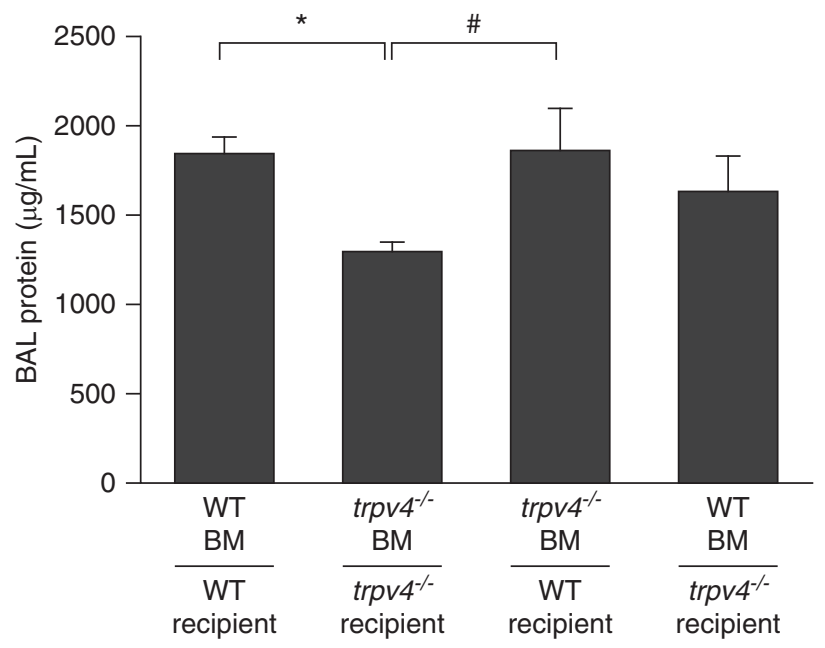

B

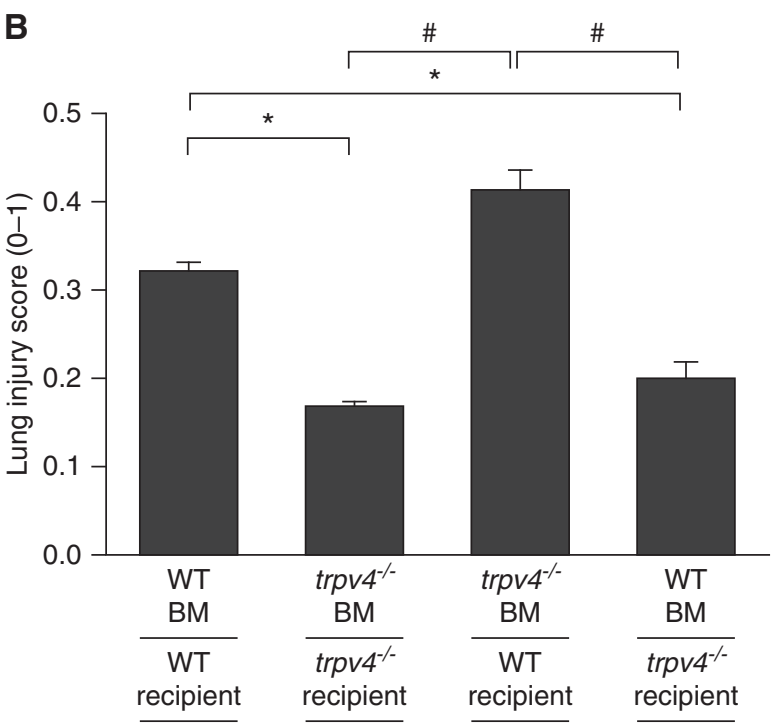

D

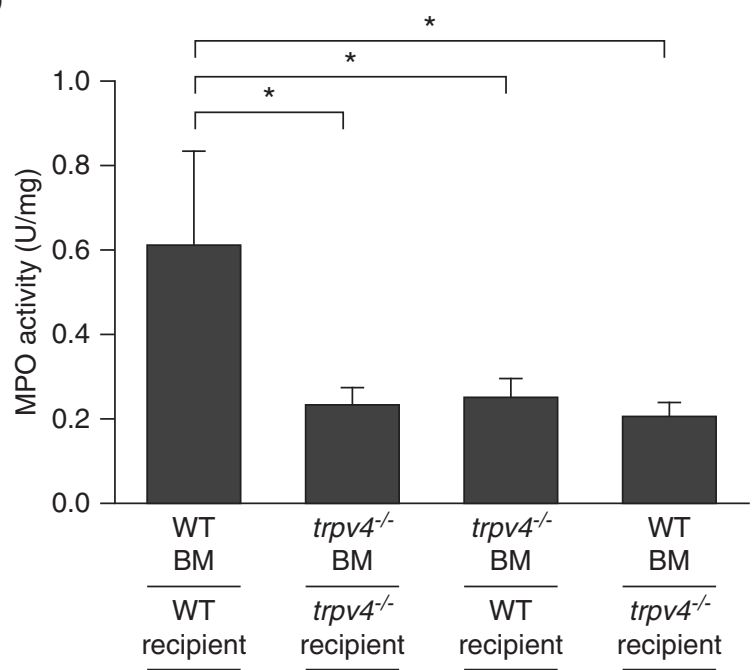

Figure 7. Role of TRPV4 in acid-induced ALI relates primarily to TRPV4 expression on lung parenchymal rather than circulating blood cells. Group data show the effects of parenchymal (recipient) or myeloid (bone marrow) TRPV4 deficiency (trpv $4^{-1-}$ ) versus WT on lung wet-to-dry-weight ratio (A), histological features of lung injury on a quantitative scale from 0 (no injury) to 1 (maximal) $(B)$, protein concentration in the BAL fluid (C), and lung MPO activity as a measure of neutrophil invasion $(D)$. Data are mean \pm SEM from $n=6$ mice per group in $A$ and $B, n=7$ in $C$ and $D$. ${ }^{*} P<0.05$ versus WT recipients with WT bone marrow $(\mathrm{BM}) ;{ }^{\#} P<0.05$ versus WT recipients with trpv $4^{-1-}$ bone marrow.

experiments in blood-perfused lungs identified an additional putative role for TRPV4 expression in circulating blood cells. The latter finding is in line with a series of studies demonstrating that microvascular leakage in ALI in vivo critically depends on the presence and function of circulating neutrophils, as vascular barrier failure and the development of a proteinaceous permeability edema are largely attenuated by neutrophil depletion or inhibition of neutrophil elastase in various models of experimental ALI (40-41).
Based on this notion, we probed for a potential functional role of TRPV4 in neutrophils that may be relevant to the pathogenesis of ALI. TRPV4 mRNA is highly expressed as compared with other channels of the transient receptor potential canonical (TRPC) or transient receptor potential melastatin (TRPM) subfamily in murine neutrophils (9) and has also been detected in human leukocytes (14); however, its relevance for neutrophil function and activation has so far not been elucidated. In the present study, we confirmed TRPV4 expression on both murine and human neutrophils at the protein level. We next demonstrated that the previously documented characteristic $\left[\mathrm{Ca}^{2+}\right]_{\mathrm{i}}$ response of neutrophils to PAF (42) is partially mediated by TRPV4 in that TRPV4 deficiency or inhibition attenuated the sustained phase of the response that is attributable to influx of extracellular $\mathrm{Ca}^{2+}$ (42). Neutrophil granulocytes have been reported to express (at least at the mRNA level) several other members of the TRP family, including TRPC6, TRPM2, TRPV1, TRPV2, TRPV5, and TRPV6, and functional roles have been reported for 
TRPM2, TRPC6 (9), TRPC1, and TRPV1. There is considerable overlap of the functional effects of different TRP channels in virtually every biological system studied so far, which has led to the recognition that different TRP channels may either be functionally or structurally coupled, or that functional TRP channels may occasionally be heteromers composed of different TRP channel subunits (43). The extent to which TRPV4 may cooperate with other TRP channels or TRP subunits in the regulation of neutrophil $\mathrm{Ca}^{2+}$ influx and downstream signaling pathways thus presents an intriguing field for further studies.

In vascular endothelial cells, TRPV4 is potently activated by EETs, in particular by the regioisomers, 5,6-EET and 8,9-EET $(11,26)$. Here, we demonstrate that TRPV4 is equally stimulated by 5,6-EET in neutrophils. PAF has previously been shown to stimulate EET formation in platelets (44), and we detected an analogous increase in various EET regioisomers in neutrophils. The obvious conclusion, that the neutrophil $\left[\mathrm{Ca}^{2+}\right]_{\mathrm{i}}$ response to PAF may be triggered by an EETdependent activation of TRPV4, was confirmed in that inhibition of EET formation by the cytochrome P450 epoxygenase inactivator, propargyloxyphenyl hexanoic acid, largely attenuated the PAF-induced increase in neutrophil $\left[\mathrm{Ca}^{2+}\right]_{\mathrm{i}}$. This result is in line with the previously reported inhibition of PAF-induced $\mathrm{Ca}^{2+}$-influx into neutrophils by various cytochrome P450 inhibitors (45), and with our present finding that the neutrophil $\left[\mathrm{Ca}^{2+}\right]_{\mathrm{i}}$ response to 5,6-EET was reduced by over $50 \%$ in trpv $4^{-1-}$ cells.

Increases in neutrophil $\left[\mathrm{Ca}^{2+}\right]_{i}$ due to $\mathrm{Ca}^{2+}$ mobilization from intracellular stores and/or influx of extracellular $\mathrm{Ca}^{2+}$ are important regulators of neutrophil function in that they are required for the effective activation of neutrophil reduced nicotinamide adenine dinucleotide phosphate oxidase and the subsequent release of superoxide anions, as well as for unimpaired neutrophil migration and chemotaxis in response to various proinflammatory stimuli. Here, we demonstrate that ROS production in response to PAF, and cell adhesion to and chemotaxis across endothelial cells, are markedly attenuated in neutrophils of $\operatorname{trp} v 4^{-l-}$ as compared with wild-type mice. This finding is in line with an important role of neutrophil $\left[\mathrm{Ca}^{2+}\right]_{i}$ signaling in these responses, and identifies TRPV4 as a key regulatory cation channel in neutrophil activation and migration. $\mathrm{Ca}^{2+}$-dependent activation of the small GTPase Rac may link these cellular responses to TRPV4mediated $\mathrm{Ca}^{2+}$ signaling, as Rac activation in response to PAF was found to be completely prevented in $\operatorname{trp} v 4^{-1-}$ neutrophils. Because both reduced nicotinamide adenine dinucleotide phosphate oxidase activation (27) and chemotaxis (29) in neutrophils essentially require activated Rac, TRPV4-dependent Rac activation may constitute a unifying master regulator of the various neutrophil responses to proinflammatory stimuli. Of note, TRPV4 may also modulate cellular responses via direct protein-protein interaction, independent from its function as $\mathrm{Ca}^{2+}$ channel (46), giving rise to the alternative or additional possibility that TRPV4-mediated $\mathrm{Ca}^{2+}$ signaling and TRPV4-dependent activation of Rac and modulation of neutrophil functions may not necessarily be causally linked. That notwithstanding, the demonstrated role of TRPV4 in Rac activation is potentially of functional relevance in ALI, as pharmacological inhibition of Rac by NSC23766 was recently shown to inhibit neutrophil transwell migration in vitro, and attenuated murine lung injury after intratracheal LPS challenge in vivo (47).

Notably, TRPV4 blockade was recently shown to protect against experimental colitis in mice (48), whereas, conversely, TRPV4 agonists triggered joint inflammation in rats (49), suggesting a potentially more generalized role of TRPV4 in inflammatory disease processes. Modulation of $\mathrm{Ca}^{2+}$ signaling and subsequent inhibition of $\mathrm{Ca}^{2+}$-dependent proinflammatory activities in neutrophils has recently gained significant interest as potential strategy to pharmacologically control autoaggressive immune reactions (50). The present identification of TRPV4 as an important regulator of neutrophil responses to proinflammatory stimuli and the recent emergence of specific pharmacological blockers of TRPV4 may therefore open new avenues for a better control of neutrophil-mediated inflammatory processes.

Although TRPV4 thus emerged as an important regulator of neutrophil activation in vitro, this role proved, however, of limited pathophysiological relevance in the murine model of acidinduced lung injury, as protection in bone marrow chimeras was largely dependent on TRPV4 deficiency on lung parenchymal cells rather than circulating blood cells. Only MPO activity was attenuated in irradiated $\operatorname{trp} v 4^{+/+}$mice reconstituted with $\operatorname{trp} v 4^{-1-}$ bone marrow, suggesting a role for TRPV4 in neutrophil adhesion and emigration in line with our in vitro findings. Our finding that parenchymal TRPV4 has a pivotal role in the pathophysiology of ALI is consistent with previous reports that had identified TRPV4 on endothelial cells as a critical regulator of lung vascular permeability (8). Along these lines, a couple of elegant studies recently demonstrated a key role for TRPV4 in the pathogenesis of cardiogenic lung edema (38) and ALI after chlorine gas inhalation (21), respectively, and the effective attenuation of these pathologies by therapeutic administration of pharmacological TRPV4 antagonists. Although TRPV4 has thus emerged as a putative therapeutic target in both hydrostatic and permeability-type lung edema, its role in these scenarios has been almost exclusively related to its expression on the pulmonary endothelium, and did not take into account a potential contribution of myeloid TRPV4. In the present study, TRPV4 on circulating blood cells effectively modulated lung vascular permeability in isolated, bloodperfused mouse lungs, yet only affected neutrophil accumulation, but not functional or histological parameters of lung injury in chimeric mice in vivo. Notably, the latter experiments were performed in the murine model of acid-induced ALI (i.e., in a scenario that is predominantly characterized by epithelial injury, and a secondary inflammatory response rather than direct activation of intravascular neutrophils [31]). Hence, although these data suggest only a minor role for neutrophilic TRPV4 in acid-induced ALI, they do not preclude a more prominent role in animal models or clinical conditions of more direct (systemic) neutrophil activation as simulated in our model of PAF 
stimulation in the isolated, bloodperfused rat lung.

Author disclosures are available with the text of this article at www.atsjournals.org.
Acknowledgments: The authors are indebted to Jean Parodo for excellent help with neutrophil isolation procedures, Julie Khang for Luminex cytokine analyses, Chris Spring for flow cytometric analyses, and Nataliya Potapova for appropriate calibration experiments (all Keenan Research Centre for Biomedical Science, St. Michael's Hospital, Toronto, ON, Canada).

\section{References}

1. Ranieri VM, Rubenfeld GD, Thompson BT, Ferguson ND, Caldwell E, Fan E, Camporota L, Slutsky AS; ARDS Definition Task Force. Acute respiratory distress syndrome: the Berlin definition. JAMA 2012;307:2526-2533.

2. Rubenfeld GD, Caldwell E, Peabody E, Weaver J, Martin DP, Neff M, Stern EJ, Hudson LD. Incidence and outcomes of acute lung injury. $N$ Engl J Med 2005;353:1685-1693.

3. Diaz JV, Brower R, Calfee CS, Matthay MA. Therapeutic strategies for severe acute lung injury. Crit Care Med 2010;38:1644-1650.

4. Ventilation with lower tidal volumes as compared with traditional tidal volumes for acute lung injury and the acute respiratory distress syndrome. The Acute Respiratory Distress Syndrome Network. N Engl J Med 2000;342:1301-1308.

5. Guérin C, Reignier J, Richard JC, Beuret P, Gacouin A, Boulain T, Mercier E, Badet M, Mercat A, Baudin O, et al.; PROSEVA Study Group. Prone positioning in severe acute respiratory distress syndrome. N Engl J Med 2013;368:2159-2168.

6. Ware LB, Matthay MA. The acute respiratory distress syndrome. N Engl J Med 2000;342:1334-1349.

7. Yin J, Kuebler WM. Mechanotransduction by TRP channels: general concepts and specific role in the vasculature. Cell Biochem Biophys 2010;56:1-18.

8. Alvarez DF, King JA, Weber D, Addison E, Liedtke W, Townsley MI. Transient receptor potential vanilloid 4-mediated disruption of the alveolar septal barrier: a novel mechanism of acute lung injury. Circ Res 2006;99:988-995.

9. Damann N, Owsianik G, Li S, Poll C, Nilius B. The calcium-conducting ion channel transient receptor potential canonical 6 is involved in macrophage inflammatory protein-2-induced migration of mouse neutrophils. Acta Physiol (Oxf) 2009;195:3-11.

10. Hamanaka K, Jian MY, Townsley MI, King JA, Liedtke W, Weber DS, Eyal FG, Clapp MM, Parker JC. TRPV4 channels augment macrophage activation and ventilator-induced lung injury. Am J Physiol Lung Cell Mol Physiol 2010;299:L353-L362.

11. Watanabe H, Vriens J, Prenen J, Droogmans G, Voets T, Nilius B. Anandamide and arachidonic acid use epoxyeicosatrienoic acids to activate TRPV4 channels. Nature 2003;424:434-438.

12. Jian MY, King JA, AI-Mehdi AB, Liedtke W, Townsley MI. High vascular pressure-induced lung injury requires $\mathrm{P} 450$ epoxygenasedependent activation of TRPV4. Am J Respir Cell Mol Biol 2008;38: 386-392.

13. Yin J, Hoffmann J, Kaestle SM, Neye N, Wang L, Baeurle J, Liedtke W, Wu S, Kuppe H, Pries AR, et al. Negative-feedback loop attenuates hydrostatic lung edema via a cGMP-dependent regulation of transient receptor potential vanilloid 4. Circ Res 2008;102:966-974.

14. Spinsanti G, Zannolli R, Panti C, Ceccarelli I, Marsili L, Bachiocco V, Frati F, Aloisi AM. Quantitative Real-Time PCR detection of TRPV1-4 gene expression in human leukocytes from healthy and hyposensitive subjects. Mol Pain 2008;4:51.

15. Link TM, Park U, Vonakis BM, Raben DM, Soloski MJ, Caterina MJ. TRPV2 has a pivotal role in macrophage particle binding and phagocytosis. Nat Immunol 2010;11:232-239.

16. Michalick L, Mertens M, Liedtke W, Kuebler WM.Transient receptor potential cation channel vanilloid (TRPV) 4 in ventilator-induced lung injury (VILI) [abstract]. FASEB J2013;27:914.12.

17. Luo Y, Dorf ME. Isolation of mouse neutrophils. Curr Protoc Immunol 2001; Chapter 3:Unit 3.20

18. Malam Z, Parodo J, Waheed F, Szaszi K, Kapus A, Marshall JC. Pre-B cell colony-enhancing factor (PBEF/Nampt/visfatin) primes neutrophils for augmented respiratory burst activity through partial assembly of the NADPH oxidase. J Immunol 2011;186: 6474-6484.
19. Waheed F, Dan Q, Amoozadeh Y, Zhang Y, Tanimura S, Speight P, Kapus A, Szászi K. Central role of the exchange factor GEF-H1 in TNF- $\alpha$-induced sequential activation of Rac, ADAM17/TACE, and RhoA in tubular epithelial cells. Mol Biol Cell 2013;24: 1068-1082.

20. Frömel T, Jungblut B, Hu J, Trouvain C, Barbosa-Sicard E, Popp R, Liebner S, Dimmeler S, Hammock BD, Fleming I. Soluble epoxide hydrolase regulates hematopoietic progenitor cell function via generation of fatty acid diols. Proc Natl Acad Sci USA 2012;109: 9995-10000.

21. Balakrishna S, Song W, Achanta S, Doran SF, Liu B, Kaelberer MM, Yu Z, Sui A, Cheung M, Leishman E, et al. TRPV4 inhibition counteracts edema and inflammation and improves pulmonary function and oxygen saturation in chemically induced acute lung injury. Am J Physiol Lung Cell Mol Physiol 2014;307:L158-L172.

22. Samapati R, Yang Y, Yin J, Stoerger C, Arenz C, Dietrich A, Gudermann $\mathrm{T}$, Adam D, Wu S, Freichel M, et al. Lung endothelial $\mathrm{Ca}^{2+}$ and permeability response to platelet-activating factor is mediated by acid sphingomyelinase and transient receptor potential classical 6 . Am J Respir Crit Care Med 2012;185:160-170.

23. Stephens CJ, Graham RM, Yadava OP, Leong LL, Sturm MJ, Taylor RR. Plasma platelet activating factor degradation and serum lipids after coronary bypass surgery. Cardiovasc Res 1992;26: 25-31.

24. Futosi K, Fodor S, Mócsai A. Neutrophil cell surface receptors and their intracellular signal transduction pathways. Int Immunopharmacol 2013;17:638-650.

25. Everaerts W, Zhen X, Ghosh D, Vriens J, Gevaert T, Gilbert JP, Hayward $\mathrm{NJ}$, McNamara CR, Xue F, Moran MM, et al. Inhibition of the cation channel TRPV4 improves bladder function in mice and rats with cyclophosphamide-induced cystitis. Proc Natl Acad Sci USA 2010;107:19084-19089.

26. Vriens J, Owsianik G, Fisslthaler B, Suzuki M, Janssens A, Voets T, Morisseau C, Hammock BD, Fleming I, Busse R, et al. Modulation of the $\mathrm{Ca}^{2}$ permeable cation channel TRPV4 by cytochrome P450 epoxygenases in vascular endothelium. Circ Res 2005;97: 908-915.

27. Kao YY, Gianni D, Bohl B, Taylor RM, Bokoch GM. Identification of a conserved Rac-binding site on NADPH oxidases supports a direct GTPase regulatory mechanism. J Biol Chem 2008;283: 12736-12746.

28. D'Souza-Schorey C, Boettner B, Van Aelst L. Rac regulates integrinmediated spreading and increased adhesion of T lymphocytes. Mol Cell Biol 1998;18:3936-3946.

29. Kuiper JW, Sun C, Magalhães MA, Glogauer M. Rac regulates Ptdlns $P_{3}$ signaling and the chemotactic compass through a redox-mediated feedback loop. Blood 2011;118:6164-6171.

30. Price LS, Langeslag M, ten Klooster JP, Hordijk PL, Jalink K, Collard JG. Calcium signaling regulates translocation and activation of Rac. J Biol Chem 2003;278:39413-39421.

31. Matute-Bello G, Frevert CW, Martin TR. Animal models of acute lung injury. Am J Physiol Lung Cell Mol Physiol 2008;295: L379-L399.

32. Matute-Bello G, Downey G, Moore BB, Groshong SD, Matthay MA, Slutsky AS, Kuebler WM; Acute Lung Injury in Animals Study Group. An official American Thoracic Society workshop report: features and measurements of experimental acute lung injury in animals. $A m \mathrm{~J}$ Respir Cell Mol Biol 2011;44:725-738.

33. Uhlig S, Goggel R, Engel S. Mechanisms of platelet-activating factor (PAF)-mediated responses in the lung. Pharmacol Rep 2005;57 (suppl):206-221.

34. Uhlig S, Yang Y, Waade J, Wittenberg C, Babendreyer A, Kuebler WM. Differential regulation of lung endothelial permeability in vitro and in situ. Cell Physiol Biochem2014;34:1-19. 
35. Garcia-Elias A, Mrkonjić S, Jung C, Pardo-Pastor C, Vicente R, Valverde MA. The TRPV4 channel. Handbook Exp Pharmacol 2014;222:293-319.

36. Nilius B, Owsianik G. Channelopathies converge on TRPV4. Nat Genet 2010;42:98-100.

37. Morty RE, Kuebler WM. TRPV4: an exciting new target to promote alveolocapillary barrier function. Am J Physiol Lung Cell Mol Physiol 2014;307:L817-L821.

38. Thorneloe KS, Cheung M, Bao W, Alsaid H, Lenhard S, Jian MY, Costell M, Maniscalco-Hauk K, Krawiec JA, Olzinski A, et al. An orally active trpv4 channel blocker prevents and resolves pulmonary edema induced by heart failure. Science Trans/ Med 2012;4:159ra148.

39. Wu S, Jian MY, Xu YC, Zhou C, Al-Mehdi AB, Liedtke W, Shin HS, Townsley Ml. $\mathrm{Ca}^{2+}$ entry via alpha1G and TRPV4 channels differentially regulates surface expression of $P$-selectin and barrier integrity in pulmonary capillary endothelium. Am J Physiol Lung Cell Mol Physiol 2009;297:L650-L657.

40. Looney MR, Su X, Van Ziffle JA, Lowell CA, Matthay MA. Neutrophils and their Fc gamma receptors are essential in a mouse model of transfusion-related acute lung injury. J Clin Invest 2006;116: 1615-1623.

41. Till GO, Johnson KJ, Kunkel R, Ward PA. Intravascular activation of complement and acute lung injury: dependency on neutrophils and toxic oxygen metabolites. J Clin Invest 1982;69:1126-1135.

42. Hauser CJ, Fekete Z, Adams JM, Garced M, Livingston DH, Deitch EA. PAF-mediated $\mathrm{Ca}^{2+}$ influx in human neutrophils occurs via storeoperated mechanisms. J Leukoc Biol 2001;69:63-68.
43. Goldenberg NM, Wang L, Ranke H, Liedtke W, Tabuchi A, Kuebler WM. TRPV4 is required for hypoxic pulmonary vasoconstriction. Anesthesiology 2015;122:1338-1348.

44. Zhu Y, Schieber EB, McGiff JC, Balazy M. Identification of arachidonate P-450 metabolites in human platelet phospholipids. Hypertension 1995;25:854-859.

45. Montero M, Garcia-Sancho J, Alvarez J. Comparative effects of cytochrome $\mathrm{P}-450$ inhibitors on $\mathrm{Ca}^{2+}$ and $\mathrm{Mn}^{2+}$ entry induced by agonists or by emptying the $\mathrm{Ca}^{2+}$ stores of human neutrophils. Biochim Biophys Acta 1993;1177:127-133.

46. Garcia-Elias A, Lorenzo IM, Vicente R, Valverde $M A$. IP 3 receptor binds to and sensitizes TRPV4 channel to osmotic stimuli via a calmodulinbinding site. J Biol Chem 2008;283:31284-31288.

47. Yao HY, Chen L, Xu C, Wang J, Chen J, Xie QM, Wu X, Yan XF. Inhibition of Rac activity alleviates lipopolysaccharide-induced acute pulmonary injury in mice. Biochimt Biophys Acta2011;1810:666-674.

48. Fichna J, Mokrowiecka A, Cygankiewicz Al, Zakrzewski PK, MaleckaPanas E, Janecka A, Krajewska WM, Storr MA. Transient receptor potential vanilloid 4 blockade protects against experimental colitis in mice: a new strategy for inflammatory bowel diseases treatment? Neurogastroenterol Motil 2012;24:e557-e560.

49. Denadai-Souza A, Martin L, de Paula MA, de Avellar MC, Muscará MN, Vergnolle N, Cenac N. Role of transient receptor potential vanilloid 4 in rat joint inflammation. Arthritis Rheum 2012;64:1848-1858.

50. Tintinger GR, Steel HC, Theron AJ, Anderson R. Pharmacological control of neutrophil-mediated inflammation: strategies targeting calcium handling by activated polymorphonuclear leukocytes. Drug Des Devel Ther 2009;2:95-104. 